Peter Bofinger

\title{
Sparen und Investieren im Spannungsfeld widerstreitender Paradigmen
}

Die Makroökonomie wird von zwei Paradigmen geprägt: dem (neo-)klassischen Modell und dem keynesianischen Modell. Da Bewegungsgesetze beider Modelle genauso diametral entgegengesetzt sind wie die des ptolemäischen und des kopernikanischen Weltbilds, muss der Versuch der Synthese zu erheblichen Widersprüchen führen (Bofinger, 2020). Das Buch „Sparen und Investieren im 21. Jahrhundert" von Carl-Christian von Weizsäcker und Hagen Krämer (2019) bietet ein interessantes Beispiel für dieses Spannungsfeld. Es analysiert die Auswirkungen der Demografie auf das Finanzsystem aus beiden Perspektiven, ohne eine eindeutige Präferenz auszusprechen. Sie begründen das damit, dass „obwohl die Wirkungsrichtung zwischen Ersparnissen und Investitionen gerade anders herum gesehen wird - die Situationsanalyse und vor allem die wirtschaftspolitischen Empfehlungen eine sehr große Schnittmenge (...) aufweisen." (v. Weizsäcker und Krämer, 2019, 212)

Auch wenn man Sympathie für die wirtschaftspolitischen Empfehlungen des Werkes hat, kann es nicht gleichgültig sein, wie die Ergebnisse hergeleitet werden, zumal bei genauerem Hinsehen deutlich wird, dass bei der Diagnose und der Therapie erhebliche Divergenzen bestehen. Der Grundgedanke und die Mehrzahl der analytischen Kapitel des Buches sind allerdings von der klassischen Weltsicht geprägt: „Wenn die Menschen mit steigender Lebenserwartung und steigendem Wohlstand ihr Vermögen schneller aufbauen wollen als ihren jährlichen Konsum, wenn aber andererseits das Produktionssystem vor einer zusätzlichen Komplexität, vor zusätzlichen Produktionsumwegen zurückschreckt, dann drückt das Kapitalangebot immer stärker auf den ,Preis'. (...) Der Preis für Kapital ist aber der Zins. Er müsste unter Bedingungen der Vollbeschäftigung ins Negative sinken, wenn es keine Gegenkraft gäbe. Diese Gegenkraft ist der Staat; (...) Der Staat muss ein negatives Kapitalangebot entfalten, indem er die Staatsschulden gerade um so viel erhöht, dass trotz der privaten Sparschwemme Vollbeschäftigung bei einem nicht-negativen Realzins erhalten bleibt.“ (v. Weizsäcker und Krämer, 2019, 9)

(c) Der/die Autor(en) 2020. Open Access: Dieser Artikel wird unter der Creative Commons Namensnennung 4.0 International Lizenz (https:// creativecommons.org/licenses/by/4.0/deed.de) veröffentlicht.

Open Access wird durch die ZBW - Leibniz-Informationszentrum Wirtschaft gefördert.

\section{Das klassische Modell des Kapitalmarkts}

Das klassische Modell überträgt die Mechanismen des Gütermarkts auf den Kapitalmarkt, der deshalb für eine Welt ohne Geld modelliert wird. Stattdessen gibt ein als „Kapital“ bezeichnetes Allzweckgut, das gleichermaBen als Konsumgut, als Investitionsgut und als Finanzmittel verwendet werden kann. So schreiben beispielsweise Barro und Sala-Martin (2004, 25): „One way to think about the one-sector technology is to an analogy with farm animals that can be eaten or used as inputs to produce more farm animals. The literature on economic growth has used more inventive examples - whith such terms as shmoohs, putty or ectoplasm - to reflect the easy transmutation of capital goods into consumables, and vice versa.“

Man kann sich die Welt der Klassik als Kornökonomie vorstellen. Spar- und Investitionsentscheidungen werden getroffen, nachdem das Korn geerntet wurde. Die privaten Haushalte entscheiden dann, wieviel sie davon konsumieren. Das nicht konsumierte Korn (Ersparnis) wird am Kapitalmarkt als „Kapital“ angeboten. Es wird von Investoren (Bauern) nachgefragt, die es aussähen. Eine Periode später werden daraus mehr Körner, sodass die Investoren den Haushalten die ausgeliehene Körnermenge plus Verzinsung zurückzahlen können. Sparen ist somit die Quelle für die Finanzierung von Investitionen.

Für von Weizsäcker und Krämer (2019) besteht die Herausforderung einer längeren Lebenserwartung und eines steigenden Wohlstands darin, dass sich die Sparneigung der privaten Haushalte erhöht und damit das Kapitalangebot. Demgegenüber trage der technische Fortschritt dazu bei, dass die Kapitalnachfrage der Unternehmen mehr oder weniger konstant bleibe.

Grafisch lässt sich dieses Problem wie folgt abbilden (vgl. Abbildung 1): Von Weizsäcker und Krämer gehen davon

Prof. Dr. Peter Bofinger ist Lehrstuhlinhaber für VWL I, Geld und internationale Wirtschaftsbeziehungen an der Universität Würzburg. 
Abbildung 1

Sparen und Realzins

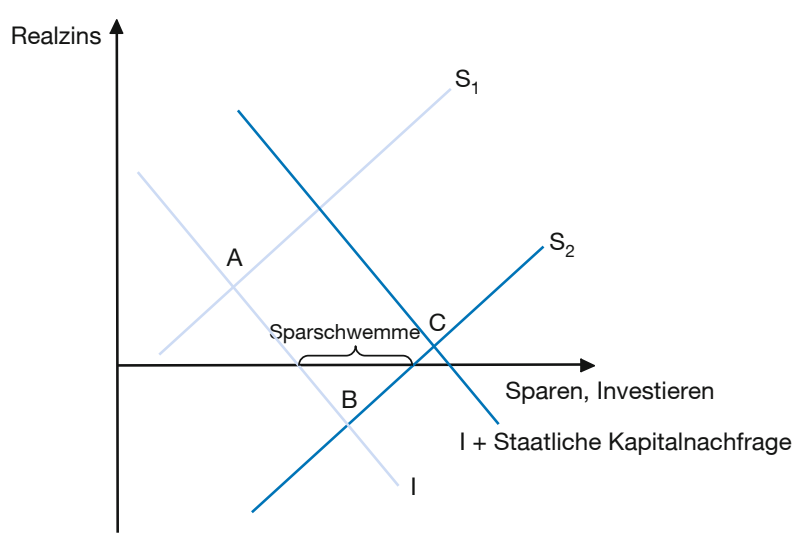

Quelle: eigene Darstellung.

aus, dass die Sparneigung (= Kapitalangebot) so stark zunimmt (Verschiebung der Sparfunktion von $S_{1}$ auf $S_{2}$ ), dass sich bei konstanter Investitionsneigung ein Gleichgewicht mit einem negativen Realzins ergibt (Punkt B). Unterstellt man, dass der Realzins nicht unter null fallen kann, ergibt sich an der Nullzins-Grenze ein Überschuss des Kapitalangebots über die Kapitalnachfrage, der oft als Sparschwemme (saving glut) bezeichnet wird.

Wenn in dieser Konstellation ein positiver Realzins erreicht werden soll, ist nach der Meinung von von Weizsäcker und Krämer der Staat gefordert, durch Staatsverschuldung ein "negatives Kapitalangebot" zu entfalten. Das ist nichts anderes als eine positive Kapitalnachfrage durch den Staat. Grafisch kommt es so zu einer Verschiebung der Kapitalnachfrage nach oben, die sich jetzt aus der privaten Investitionsnachfrage und der staatlichen Kapitalnachfrage zusammensetzt. Dies ermöglicht ein Gleichgewicht von Kapitalangebot und Kapitalnachfrage bei einem positiven Realzins (Punkt C). Bei dieser mechanischen Darstellung stellt sich die Frage, ob überhaupt ein wirtschaftspolitischer Handlungsbedarf besteht. Vollbeschäftigung ist in diesem Modell stets gewährleistet. Da es keine Zielwerte für den Realzins gibt, ist dessen Rückgang kein offensichtlicher Grund für ein staatliches Eingreifen.

Selbst bei einer Sparschwemme ist zu prüfen, ob die Staatsverschuldung (negative Kapitalnachfrage) eine Problemlösung bietet. Das erforderte, dass der Staat das von inm nachgefragte Allzweckgut investiv verwenden und damit einen positiven Realzins erwirtschaften kann. Wenn solche technologischen Möglichkeiten existierten, wäre zu fragen, warum sie nicht von privaten In- vestoren genutzt werden. Ohne eine produktive Investitionsmöglichkeit gibt es in der Folgeperiode keine zusätzlichen Einheiten des Allzweckgutes. Der Staat wäre nicht in der Lage, den Privaten einen positiven Realzins zu zahlen. Die Staatsverschuldung führte zum Staatsbankrott.

Von Weizsäcker und Krämer $(2019,226)$ setzen sich damit nicht auseinander. Sie stellen lapidar fest: „Wir konzentrieren uns in diesem Buch auf die Darstellung des Befunds eines Sparüberschusses, seine theoretische Herleitung und Einordnung sowie auf seine empirische Unterfütterung. (...). Wofür zusätzlich entstehende Staatseinnahmen verwendet werden, ist in diesem $\mathrm{Zu}$ sammenhang zweitrangig und nicht Gegenstand dieses Buches."

\section{Das keynesianische Modell des Kapitalmarktes}

Das keynesianische Modell unterscheidet sich vom klassischen Modell dadurch, dass es anstelle des Einheitsgutes mit drei Assets operiert: Geld (d.h. Sichtguthaben bei Banken), einem Konsumgut und einem Investitionsgut. Von Weizsäcker und Krämer $(2019,225)$ diskutieren das Problem der Demografie sehr ausführlich auch aus keynesianischer Sicht. Sie weisen darauf hin, wie unterschiedlich die Funktionsmechanismen der beiden Paradigmen sind: „Im keynesianischen Analyserahmen setzen die Ersparnisse der privaten Haushalte prinzipiell keine Mittel für Investitionen frei. Ein Anstieg der Ersparnisse verteilt lediglich bereits existierende Geldmittel um, hier z. B. von den Unternehmen zu den privaten Haushalten. Im Aggregat bleiben die liquiden Mittel hierdurch unverändert, weshalb auch der Zins auf das erhöhte Sparen des Haushaltssektors nicht reagieren wird."

Allerdings stört es sie nicht, dass damit dem zentralen Argument ihres Werkes der Boden entzogen wird. Wenn das Sparen der Haushalte keine finanziellen Mittel freisetzt, gibt es auch kein zusätzliches Angebot am Kapitalmarkt, das zu sinkenden Zinsen führt und eine höhere Staatsverschuldung erforderlich werden lässt. Ein Ungleichgewicht $(\mathrm{S}>\mathrm{I})$ ist in diesem Modell kein Überschussangebot an Kapital (Sparschwemme), sondern ein Überschussangebot an Konsumgütern, das zu einer unfreiwilligen Lagerbildung führt.

Von Weizsäcker und Krämer $(2019,225)$ nehmen das nicht zum Anlass, die klassische Modelllogik zu hinterfragen. Vielmehr betonen sie die Vereinbarkeit beider Ansätze: „Während also im keynesianischen Denkmodell der Zins aus grundsätzlichen Erwägungen nicht dafür infrage kommt, ein Ungleichgewicht zwischen Sparen und Investieren abzubauen, kann im hier vorgestellten neuen 
neoklassischen Denkmodell der Zins unter den gegebenen Bedingungen des 21. Jahrhunderts diese Funktion faktisch nicht mehr erfüllen.“

Dabei übersehen sie, dass das Ungleichgewicht im keynesianischen Modell durch eine Kontraktion des Einkommens abgebaut wird. Wenn das System im Gleichgewicht war, ergibt sich - im Gegensatz zum klassischen Modell - aus einer höheren Sparneigung grundsätzlich ein wirtschaftspolitischer Handlungsbedarf.

Dabei ist es jedoch nicht zwingend notwendig, das Gleichgewicht durch eine höhere Staatsverschuldung wiederherzustellen. Der Rückgang des Einkommens kann auch durch eine expansive Geldpolitik kompensiert werden. Während es in der klassischen Modellwelt keine Handlungsmöglichkeit für die Notenbank gibt, da sie das Allzweckgut weder produzieren noch konsumieren kann, stellt sie im keynesianischen Modell den wichtigsten Akteur dar. ${ }^{1}$ Im keynesianischen Modell muss der Staat erst dann mit höheren Ausgaben und geringeren Steuern aktiv werden, wenn die Notenbank an den Nullzinsgrenze gerät.

Natürlich kann der Staat in diesem Modellrahmen durch kreditfinanzierte staatliche Investitionen eine unzureichende gesamtwirtschaftliche Nachfrage kompensieren. Er absorbiert dabei aber keine überschüssige Ersparnis. Vielmehr generieren seine Investitionen über den Einkommensmultiplikator zusätzliche Erspanis.

\section{Der empirische Befund}

Eine zentrale Annahme des Buches besteht darin, dass es aufgrund der Demografie und eines steigenden Wohlstands zu einem säkularen Anstieg der Sparquote der privaten Haushalte komme. „(...) die Sparquote (freiwillige und erzwungene zusammengenommen) der Berufstätigen wird mit steigender Lebenserwartung weiter steigen.“ (v. Weizsäcker und Krämer, 2019, 6)

Diese Annahme prägt viele Studien, die den Rückgang der Realzinsen seit Anfang der 1980er Jahre analysieren (Rachel und Smith, 2017). Sie ist jedoch mit der empirischen Evidenz nicht vereinbar, da die Sparquoten der privaten Haushalte seit dieser Zeit in den fortgeschrittenen Volkswirtschaften teilweise drastisch zurückgegangen sind (vgl. Abbildung 2).

1 Von Weizsäcker und Krämer sprechen die Möglichkeit an. Sie weisen ihr jedoch keine größere Bedeutung zu: „Da die Geldpolitik hier schnell an ihre Grenzen stößt, kommen im Wesentlichen nur fiskalische Maßnahmen infrage." (v. Weizsäcker und Krämer, 2019, 215)

\section{Abbildung 2}

Netto-Sparquoten ${ }^{1}$ der privaten Haushalte

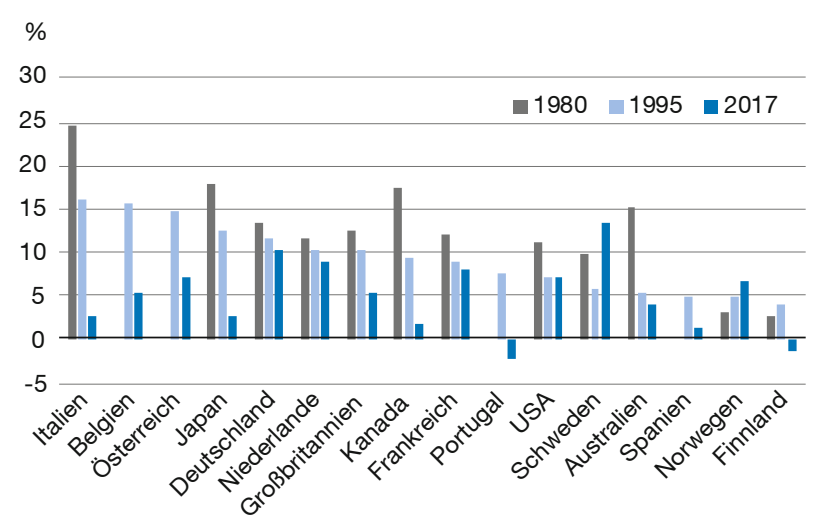

${ }^{1}$ Für Großbritannien wurde die Brutto-Sparquote verwendet.

Anmerkungen: Für Deutschland ist der Wert für 1980 für Westdeutschland. Für einige Länder waren keine Daten für 1980 verfügbar. Für Kanada war erst ein Wert für 1981 verfügbar.

Quelle: OECD, AMECO und Office for National Statistics.

Man könnte diesem Befund entgegenhalten, dass die Sparneigung zwar zugenommen habe, sich aber bei einem gleichzeitigen Rückgang der Investitionsneigung im Gleichgewicht keine höhere Sparquote eingestellt habe. Bei den ausgeprägten Rückgängen ist das unwahrscheinlich. Eine plausible Ursache für diese Entwicklung besteht darin, dass sich die funktionale Einkommensverteilung in den vergangenen Jahrzehnten gravierend zulasten des Faktors Arbeit verändert hat (vgl. Abbildung 3). So gesehen hätte für die Arbeitnehmer zwar die Notwendigkeit bestanden, stärker für das Alter zu sparen. Aufgrund ihrer ungünstigeren Einkommensposition waren sie jedoch nicht in der Lage, diese Pläne auch umzusetzen. Damit fällt die empirische Basis bei von Weizsäcker und Krämer ebenso in sich zusammen wie die gängigen Erklärungsansätze für niedrige Zinsen.

Wie kann der Staat die mit der Demografie verbundenen Probleme angehen?

Das Denken in der klassischen Modellwelt verleitet somit zu dem Fehlschluss, dass sinkende Zinsen auf eine steigende Sparneigung der privaten Haushalte zurückzuführen seien. Dabei ist das Problem genau umgekehrt. Anstelle einer Sparschwemme ist es einkommensbedingt zu einem unzureichenden Sparen der privaten Haushalte gekommen. 
Abbildung 3

Veränderung der bereinigten Lohnquote (2016 bis 2018 gegenüber 1978 bis 1980)

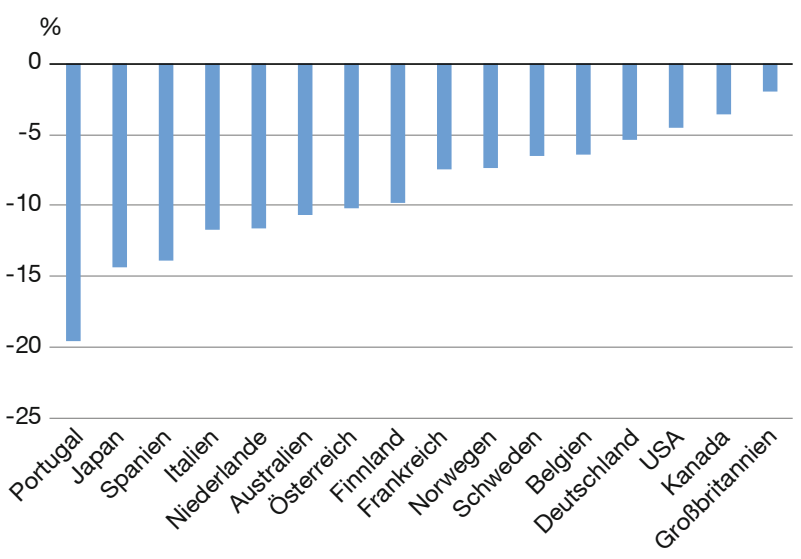

Anmerkungen: Um den Einfluss der Konjunktur auf die Lohnquote zu vermindern, wurden Dreijahresdurchschnitte ermittelt.

Quelle: AMECO.

Wie kann der Staat dann dazu beitragen die Altersvorsorge der Bevölkerung zu verbessern? Aus der Perspektive des keynesianischen Modells ist das Umlagesystem jeder Form der kapitalgedeckten Altersvorsorge überlegen. Es ermöglicht den Arbeitnehmern, in der Zukunft ein Einkommen zu sichern, ohne dass es dafür in der Gegenwart zu einem gesamtwirtschaftlichen Konsumverzicht kommen muss.

Bei von Weizsäcker und $\operatorname{Krämer}(2019,198)$ spielt das Umlagesystem eine wichtige Rolle, da die Autoren die daraus erwachsenden zukünftigen Zahlungen des Staates als Staatsverschuldung klassifizieren: „Nach unseren Schätzungen machte die implizite Staatsverschuldung in der Region OECD plus China in 2015 das 5,39-fache des volkswirtschaftlichen Jahreskonsums aus. Die implizite Staatsverschuldung ist daher viel bedeutsamer für die Vermögensansprüche der Privaten gegenüber dem Staat. Der mit Abstand größte Teil stammt hierbei aus den staatlichen Rentenversicherungen, für den wir in 2015 eine implizite Staatsverschuldung von 482 Prozent des volkswirtschaftlichen Konsums in der Region OECD plus China kalkuliert haben.“

Gleichzeitig betrachten von Weizsäcker und Krämer $(2019,4)$ die den Privaten aus dem Umlagesystem in der Zukunft erwachsenden Einnahmen als einen zentralen Bestandteil des Privatvermögens: „Für den Bürger ist das Anrecht auf diese künftigen Renten- und Pensionszahlungen jedoch ohne Zweifel Vermögen. Seine
Lebensplanung, insbesondere sein gegenwärtiges Konsumverhalten richtet sich auf diese künftig zu erwartenden Zahlungen ein.“

Bei dieser Betrachtungsweise werden zwar die aus dem Umlagesystem vom Staat an die Privaten zu leistenden Zahlungen berücksichtigt. Die gleichzeitig von den Privaten an den Staat zu leistenden Beiträge für die Rentenversicherung werden jedoch ausgeblendet. Wenn man implizite Staatsschulden ausweist, sollte man auch die impliziten Staatsforderungen berücksichtigen. Aus der Sicht eines individuellen Haushalts ist es zutreffend, die Rentenansprüche als Teil des Vermögens zu betrachten. Für den Privatsektor insgesamt sind die bei einem Umlagesystem von ihm zu leistenden Zahlungen zu jedem Zeitpunkt identisch mit den von inm empfangenen Renten. Damit entsprechen sich die Barwerte der beiden Zahlungsströme.

Für das Umlagesystem stellt eine längere Lebenserwartung kein Problem dar, wenn die zusätzlichen Lebensjahre gleichzeitig mit einer höheren Leistungsfähigkeit einhergehen. Das Renteneintrittsalter kann dann entsprechend erhöht werden, wie dies in Deutschland durch die Rentenreform des Jahres 2007 geschehen ist. Anders als bei von Weizsäcker und Krämer unterstellt ${ }^{2}$ muss dabei die Arbeitszeit nicht in demselben Maße ansteigen wie das Lebensalter.

Aus der Logik des Umlagesystems lässt sich die Rolle der Staatsverschuldung schlüssig ableiten. Wenn aus diesem System in der Zukunft Rentenzahlungen geleistet werden sollen, die den Wohlstand der Rentner sichern, muss der Staat dafür sorgen, dass sich die Leistungsfähigkeit der Wirtschaft verbessert. Das erfordert vor allem, dass die Beschäftigten über ein möglichst hohes Bildungsniveau verfügen. Diesem Aspekt wird bei von Weizsäcker und Krämer keine größere Beachtung geschenkt: „Eine volkswirtschaftlich wichtige Vermögensform behandeln wir nicht: das Humankapital. Der Grund liegt darin, dass es, von wenigen Ausnahmen abgesehen, nicht als Kapitalanlageobjekt für Dritte zur Verfügung steht.“ (v. Weizsäcker und Krämer, 2019, 57)

Zudem kommt der Infrastruktur eine entscheidende Bedeutung für die Wettbewerbsfähigkeit einer Volkswirtschaft zu. Es ist daher alles andere als „zweitrangig“ ( $v$. Weizsäcker und Krämer, 2019, 226) wofür der Staat die Mittel ausgibt, die er am Kapitalmarkt aufnimmt. Es gibt

2 „Der Staat hat auch wenig andere Möglichkeiten, darauf zu reagieren, solange seine Bürger ihre längere Lebenszeit nicht in eine gleich groBe Verlängerung ihrer Lebensarbeitszeit umsetzen wollen." (v. Weizsäcker und Krämer, 2019, 225). 
also aus der Sicht des Umlagesystems gute Argumente für eine höhere Staatsverschuldung, die die Produktivität der Volkswirtschaft stärkt. So kann dem Vermögenswunsch einer alternden Bevölkerung effektiv Rechnung getragen werden.

\section{Zusammenfassung}

Von Weizsäcker und Krämer diagnostizieren eine „private Sparschwemme“, für die sie als Therapie ein „negatives Kapitalangebot" des Staates vorschlagen. Im Rahmen des klassischen Modells stellt sich die Frage, ob es sich dabei überhaupt um eine Fehlentwicklung handelt. Im Modell herrscht stets Vollbeschäftigung. Sinkende Zinsen bedingen grundsätzliche keine staatliche Intervention. Sie sind vielmehr die Marktantwort auf ein Ungleichgewicht zwischen Kapitalangebot und Kapitalnachfrage. In der Welt des Allzweckgutes kann die Staatsverschuldung zudem keinen effektiven Beitrag für höhere Zinsen leisten. Bei einer technologisch determinierten Kapitalnachfrage müsste der Staat über Produktionsverfahren für den Einsatz des Einheitsgutes verfügen, die den Privaten nicht zugänglich sind.

Im keynesianischen Modell führt die erhöhte Sparneigung der privaten Haushalte dazu, dass die gesamtwirtschaftliche Nachfrage sinkt. Anstelle eines Überangebots an Kapital besteht ein Überangebot an Konsumgütern, das zu einem Rückgang der gesamtwirtschaftlichen Nachfrage führt. Damit ist das keynesianische Modell unvereinbar mit dem zentralen Argument von Von Weizsäcker und Krämer: dem Sparüberschuss aufgrund eines erhöhten Kapitalangebots. Als wirtschaftspolitische Antwort ist eine höhere Staatsverschuldung nicht zwingend, da die Notenbank jederzeit zinspolitisch aktiv werden kann. Natürlich kann das Ungleichgewicht am Gütermarkt über schuldenfinanzierte Staatsausgaben kompensiert werden. Damit wird jedoch keine Sparschwemme abgesaugt, sondern über das erhöhte Einkommen zusätzliche Ersparnis generiert.

Schließlich gibt es keine empirische Evidenz für eine steigende Ersparnis der privaten Haushalte. Aufgrund der sinkenden Lohnquote ist es wahrscheinlich vielen Haushalten nicht möglich gewesen, für ihr Alter finanziell vorzusorgen. Kritisch anzumerken ist zudem, dass von Weizsäcker und Krämer die implizite Staatsverschuldung aus dem Umlagesystem als Vermögen des privaten Sektors betrachten. Da in diesem System sich zu jedem Zeitpunkt die Einzahlungen durch die Privaten und die Auszahlungen an die Privaten entsprechen sollten, ist der Barwert des Ganzen gleich null.
Tabelle 1

Klassisches versus keynesianisches Paradigma im Vergleich

\begin{tabular}{|c|c|c|}
\hline & $\begin{array}{l}\text { Klassisches } \\
\text { Paradigma }\end{array}$ & $\begin{array}{l}\text { Keynesianisches } \\
\text { Paradigma }\end{array}$ \\
\hline Assetklassen & Allzweckgut & $\begin{array}{l}\text { Geld, Konsumgut, } \\
\text { Investitionsgut }\end{array}$ \\
\hline$S>1$ & $\begin{array}{l}\text { Überschussangebot an } \\
\text { Kapital (Sparschwemme) }\end{array}$ & $\begin{array}{l}\text { Überschussangebot } \\
\text { an Konsumgütern }\end{array}$ \\
\hline $\begin{array}{l}\text { Primäreffekt einer } \\
\text { höheren Sparneigung }\end{array}$ & $\begin{array}{l}\text { Realzins sinkt (kein Effekt } \\
\text { auf Beschäftigung) }\end{array}$ & $\begin{array}{l}\text { Einkommen und } \\
\text { Beschäftigung sin- } \\
\text { ken (kein Effekt auf } \\
\text { Geldzins) }\end{array}$ \\
\hline $\begin{array}{l}\text { Wirtschaftspolitischer } \\
\text { Handlungsbedarf }\end{array}$ & $\begin{array}{l}\text { Unklar: Realzins ist } \\
\text { Marktergebnis }\end{array}$ & $\begin{array}{l}\text { Eindeutig: Arbeitslo- } \\
\text { sigkeit }\end{array}$ \\
\hline $\begin{array}{l}\text { Effekt der Staats- } \\
\text { verschuldung }\end{array}$ & $\begin{array}{l}\text { Absorbiert Ersparnis. } \\
\text { Positiver Effekt auf Re- } \\
\text { alzins nur bei rentablen } \\
\text { Investitionen }\end{array}$ & $\begin{array}{l}\text { Generiert Erspar- } \\
\text { nis. Positiver Effekt } \\
\text { auf Beschäftigung } \\
\text { immer gegeben }\end{array}$ \\
\hline
\end{tabular}

Quelle: eigene Darstellung.

Insgesamt verdeutlicht das Werk, wie widersprüchlich beide Paradigmen sind (vgl. Tabelle 1) und welche Probleme sich stellen, wenn man das klassische Modell auf die Realität anwenden will. Keynes hat das so formuliert: „The characteristics of the special case assumed by the classical theory happen not to be those of the economic society in which we actually live, with the result that its teaching is misleading and disastrous if we attempt to apply it to the facts of experience." (Keynes, 1936 [1973], 3)

\section{Literatur}

Barro. R. und X. Sala-i-Martin (2004), Economic Growth, Second Edition, MIT Press.

Bofinger, P. (2020), Reviving Keynesianism: the modelling of the financial system makes the difference, Review of Keynesian Economics, 8(1), 61-83.

Keynes, J. M. (1936), The General Theory of Employment, Interest, and Money, in J. M. Keynes (1973), The Collected Writings, Vol. 7, Macmillan.

Rachel, L. und T. D. Smith (2017), Are low real interest rates here to stay?, International Journal of Central Banking, 13(3), 1-42.

v. Weizsäcker, C. C. und H. Krämer (2019), Sparen und Investieren im 21. Jahrhundert. Die große Divergenz, Springer. 